\title{
Correction to: Soluble delta-like 1 homolog (DLK1) stimulates angiogenesis through Notch1/Akt/eNOS signaling in endothelial cells
}

\author{
Chao-Cheng Huang ${ }^{1,2} \cdot$ Hsiao-Mei Kuo ${ }^{3,4} \cdot$ Pei-Chang Wu ${ }^{5}$. Shih-Hsuan Cheng ${ }^{1,11}$. Tzu-Ting Chang 4,6 . \\ $\mathrm{Yi}^{\mathrm{i}-C h e n} \mathrm{Chang}^{7,8} \cdot$ Mei-Lang Kung $^{9} \cdot$ Deng-Chyang Wu${ }^{10,11} \cdot$ Jiin-Haur Chuang ${ }^{12} \cdot$ Ming-Hong Tai ${ }^{3,4,7,8,10,11,13}$
}

Published online: 10 May 2018

(c) Springer Science+Business Media B.V., part of Springer Nature 2018

\section{Correction to: Angiogenesis (2018) 21:299-312 https://doi.org/10.1007/s10456-018-9596-7}

In the original publication of the article, there is an error in one of the citations in the Discussion section. The citation of the article by Jensen et al. [9] on the protein structure of fetal antigen-1 is clearly a mistake in the context of the Discussion text on the potential for DLK1 to act as an angiogenesis inhibitor.

The correct reference to be cited in the Discussion section at the end of the sentence: "However, there was also conflicting view that DLK1 acts as an angiogenesis inhibitor from studies using DLK1-null mice and zebrafish models" is given below.

\section{Reference}

Rodríguez P, Higueras MA, González-Rajal A, Alfranca A, Fierro-Fernández M, García-Fernández RA, Ruiz-Hidalgo MJ, Monsalve M, Rodríguez-Pascual F, Redondo JM, de la Pompa JL, Laborda J, Lamas S (2012) The non-canonical NOTCH ligand DLK1 exhibits a novel vascular role as a strong inhibitor of angiogenesis. Cardiovasc Res 93(2):232241. https://doi.org/10.1093/cvr/cvr296

7 Doctoral Degree Program in Marine Biotechnology, National Sun Yat-Sen University, Kaohsiung, Taiwan

8 Doctoral Degree Program in Marine Biotechnology, Academia Sinica, Taipei, Taiwan

9 Department of Chemistry, National Sun Yat-Sen University, Kaohsiung, Taiwan

10 Center for Stem Cell Research, Department of Internal Medicine, Kaohsiung Medical University, Kaohsiung, Taiwan

11 Division of Gastroenterology, Department of Internal Medicine, Kaohsiung Medical University, Kaohsiung, Taiwan

12 Division of Pediatric Surgery, Department of Surgery, Kaohsiung Chang Gung Memorial Hospital, Kaohsiung 833, Taiwan

13 Institute of Biomedical Sciences, National Sun Yat-Sen University, 70 Lien-Hai Rd, Kaohsiung 804, Taiwan 